\title{
Up-gradient eddy fluxes of potential vorticity near the subtropical jet
}

Article

Published Version

Birner, T., Thompson, D. W. J. and Shepherd, T. G. (2013) Upgradient eddy fluxes of potential vorticity near the subtropical jet. Geophysical Research Letters, 40 (22). pp. 5988-5993. ISSN 0094-8276 doi: https://doi.org/10.1002/2013GL057728 Available at https://centaur.reading.ac.uk/35573/

It is advisable to refer to the publisher's version if you intend to cite from the work. See Guidance on citing.

To link to this article DOI: http://dx.doi.org/10.1002/2013GL057728

Publisher: American Geophysical Union

All outputs in CentAUR are protected by Intellectual Property Rights law, including copyright law. Copyright and IPR is retained by the creators or other copyright holders. Terms and conditions for use of this material are defined in the End User Agreement.

\section{www.reading.ac.uk/centaur}

\section{CentAUR}

Central Archive at the University of Reading

Reading's research outputs online 


\title{
Up-gradient eddy fluxes of potential vorticity near the subtropical jet
}

\author{
T. Birner, ${ }^{1}$ D. W. J. Thompson, ${ }^{1}$ and T. G. Shepherd ${ }^{2}$ \\ Received 22 August 2013; revised 28 October 2013; accepted 3 November 2013; published 19 November 2013.
}

[1] The role of eddy fluxes in the general circulation is often approached by treating eddies as (macro)turbulence. In this approach, the eddies act to diffuse certain quasiconservative quantities, such as potential vorticity (PV), along isentropic surfaces in the free atmosphere. The eddy fluxes are determined primarily by the eddy diffusivities and are necessarily down-gradient of the basic state PV field. Support for the (macro)turbulence approach stems from the fact that the eddy fluxes of PV in the free atmosphere are generally down-gradient in the long-term mean. Here we call attention to a pronounced and significant region of upgradient eddy PV fluxes on the poleward flank of the jet core in both hemispheres. The region of up-gradient (i.e., notionally "antidiffusive") eddy PV fluxes is most pronounced during the winter and spring seasons and partially contradicts the turbulence approach described above. Analyses of the PV variance (potential enstrophy) budget suggest that the up-gradient PV fluxes represent local wave decay and are maintained by poleward fluxes of PV variance. Finite-amplitude effects thus represent leading order contributions to the PV variance budget, whereas dissipation is only of secondary importance locally. The appearance of up-gradient PV fluxes in the long-term mean is associated with the poleward shift of the jet-and thus the region of wave decay relative to wave growth - following wave-breaking events. Citation: Birner, T., D. W. J. Thompson, and T. G. Shepherd (2013), Up-gradient eddy fluxes of potential vorticity near the subtropical jet, Geophys. Res. Lett., 40, 5988-5993, doi:10.1002/2013GL057728.

\section{Eddies and the General Circulation}

[2] Eddies play a dominant role in determining the general circulation of the extratropical atmosphere. The role of the eddies is often discussed from a macroturbulence perspective [Held, 1999], which assumes that eddies act to diffuse certain quasi-conserved quantities. The diffusive nature of the eddies was recognized at least as early as Taylor [1914], Defant [1921], and Jeffreys [1926]. For example, Defant stated that "The idea that the midlatitude flow can be regarded as pronounced grand-scale turbulence should not be viewed all too venturous" (loosely translated from German).

[3] The underlying assumption for the macroturbulent picture to work is essentially that the transport/eddy length

\footnotetext{
${ }^{1}$ Department of Atmospheric Science, Colorado State University, Fort Collins, Colorado, USA.

${ }^{2}$ Department of Meteorology, University of Reading, Reading, UK.
}

Corresponding author: T. Birner, Department of Atmospheric Science, Colorado State University, 1371 Campus Delivery, Fort Collins, CO 805231371, USA. (thomas@atmos.colostate.edu)

(C)2013. American Geophysical Union. All Rights Reserved. 0094-8276/13/10.1002/2013GL057728 scales are small compared to the size of the turbulent region [e.g., Held, 2000; Sobel, 1999]. Even though this assumption may be questionable for the Earth's atmosphere, the diffusive picture of eddy fluxes has been applied with success to potential vorticity (PV) being fluxed along isentropic surfaces in the free atmosphere [e.g., Green, 1970; McIntyre, 2000; Schneider, 2004; Butler et al., 2011]. Based on this picture, eddies will tend to flatten the background isentropic gradients of PV, i.e., they will tend to homogenize PV along isentropic surfaces [e.g., Rhines and Young, 1982].

[4] A refinement of the macroturbulent viewpoint arises by treating eddies as (Rossby) waves. PV again plays a fundamental role in that its meridional gradient along isentropic surfaces determines wave propagation characteristics. For example, the refractive index for Rossby waves is largely a function of the meridional PV gradient. Regions of strong meridional PV gradient, such as at the core of jet streams, represent wave guides, i.e., they favor wave propagation. Hence, macroturbulence in the free atmosphere should be understood in terms of Rossby wave breaking [e.g., Held and Hoskins, 1985; Randel and Held, 1991] organized by wave guides, which necessarily makes it spatially inhomogeneous and can lead to sharpened PV gradients [Haynes et al., 2001]. McIntyre [2008] points out that eddies need to be viewed from both a wave-like and a turbulent perspective.

[5] The question may be asked: To what extent is the diffusive picture of down-gradient eddy PV fluxes (locally) valid in the real atmosphere? Potential violations of this picture (or the lack thereof) may provide important insights into the role of eddies in the general circulation, including implications for eddy-mean flow feedback processes which are crucial for our understanding of large-scale climate variability and long-term climate change [e.g., Robinson, 2000; Lorenz and Hartmann, 2001; Thompson and Birner, 2012]. Here we present an analysis of eddy PV fluxes in the troposphere and lowermost stratosphere based on modern reanalysis data, which reveals a localized region of significant up-gradient (notionally antidiffusive) eddy PV fluxes on the poleward flank of the subtropical jet core during the winter and spring seasons of both hemispheres. Based on a detailed analysis of the potential enstrophy ( $\sim$ PV variance) budget, we formulate a plausible mechanism for the up-gradient PV fluxes, involving characteristics of wave breaking and decay along the subtropical jet, together with poleward migration of the jet following wave-breaking events.

\section{Diffusive (Down-Gradient) Eddy Fluxes of Potential Vorticity}

[6] Atmospheric large-scale dynamics may be best described through the evolution of the PV field along isentropic surfaces [Hoskins et al., 1985]. In quasi-geostrophic (QG) theory, the dynamics are fully governed by QG PV 
(as well as potential temperature on rigid boundaries). The background potential temperature field is assumed to be horizontally homogeneous, such that isentropes coincide with constant pressure surfaces. For the sake of simplicity of the notation, we will discuss all following relations in a quasi-geostrophic (QG) framework in Cartesian coordinates, generalizing the final relations to the primitive equations in isentropic coordinates.

[7] The (macroturbulent) diffusive picture of the role of eddies in the general circulation results in down-gradient eddy fluxes of PV, with a formal flux-gradient relationship of the form [e.g., Vallis, 2006, chapter 12.7]:

$$
\overline{v^{\prime} q^{\prime}}=-\mathcal{D} \partial_{y} \bar{q},
$$

where $q$ is the QG PV, $\mathcal{D}$ is the eddy diffusivity, and overbars denote zonal averages, with primes denoting deviations therefrom.

[8] In the Transformed Eulerian Mean framework [e.g., Andrews et al., 1987], the meridional eddy PV flux formally corresponds to the wave stress (the divergence of the Eliassen-Palm flux) and acts as the main eddy forcing term in the zonal mean momentum budget

$$
\partial_{t} \bar{u}-f_{0} \bar{v}^{*}=\overline{v^{\prime} q^{\prime}}
$$

Here $f_{0}$ is the Coriolis parameter, and $\bar{v}^{*}$ is the meridional residual velocity. The flux-gradient relation for the eddy PV flux therefore provides a closure for the zonal mean momentum budget. It also provides an explanation for the predominantly poleward residual circulation in the extratropical free atmosphere: In steady state, the flux-gradient relation yields $f_{0} \bar{v}^{*} \simeq \mathcal{D} \partial_{y} \bar{q}$, that is, $\bar{v}^{*}$ has the same sign as $f_{0}$ for $\partial_{y} \bar{q}>0$ and positive definite $\mathcal{D}$. In particular, for positive background meridional PV gradient $\left(\partial_{y} \bar{q}>0\right)$, the resulting forcing given by the eddy PV flux is always negative, i.e., wave stress corresponds to wave drag (converging Eliassen-Palm flux) and $\bar{v}^{*}$ is poleward.

[9] The flux-gradient relation is more formally based on the PV variance-potential enstrophy (hereafter referred to as "enstrophy" for simplicity) - equation, related to wave activity [e.g., Vallis, 2006]:

$$
\partial_{t} \frac{\overline{q^{\prime 2}}}{2}=\overline{S^{\prime} q^{\prime}}-\overline{v^{\prime} q^{\prime}} \partial_{y} \bar{q}+\mathcal{O}(3)
$$

with $S$ denoting sources and sinks of QG PV, and $\mathcal{O}(3)$ denoting terms of third or higher order (such as the convergence of eddy fluxes of PV variance; see further below). Neglecting these higher order nonlinear terms results in the simple steady state balance

$$
\overline{v^{\prime} q^{\prime}} \partial_{y} \bar{q}=\overline{S^{\prime} q^{\prime}}
$$

That is, for dissipative processes (more precisely processes that dissipate enstrophy, e.g., $S^{\prime} \simeq-\alpha q^{\prime}$, with inverse damping rate $\alpha>0$; see e.g., Plumb [1979] for discussion) in steady state [e.g., Andrews, 1983a],

$$
\alpha \overline{q^{\prime 2}} \simeq \overline{-v^{\prime} q^{\prime}} \partial_{y} \bar{q}
$$

Given that the left-hand side is always positive, the eddy PV flux has to be down-gradient (of opposite sign to the background PV gradient) for this relation to hold, lending credibility to the diffusive picture of eddy PV fluxes.
[10] According to equation (1), down-gradient eddy PV fluxes correspond to eddy/wave growth (positive $\overline{q^{\prime 2}}$ tendency), while eddy/wave decay may temporarily produce up-gradient eddy PV fluxes (see also discussion in Plumb [1979] and Hoskins [1983]). For reversible growth and decay, the up-gradient eddy PV fluxes exactly cancel the down-gradient fluxes, resulting in zero net eddy PV flux (fulfilling the nonacceleration conditions). However, if wave breaking is involved, the up-gradient eddy PV fluxes during wave decay will be smaller in magnitude than the downgradient eddy PV fluxes during wave growth, resulting in net down-gradient eddy PV fluxes. This is consistent with the above simple steady state balance, under which only the dissipative term $\left(\overline{S^{\prime} q^{\prime}}\right)$ represents negative $\overline{q^{\prime 2}}$ tendencies. In steady state, up-gradient eddy PV fluxes would be in conflict with the simple (second order) balance above and would violate the flux-gradient relation of the diffusive picture with $\mathcal{D}>0$ (and notionally correspond to antidiffusion, i.e., negative diffusivity).

[11] By analyzing the enstrophy tendency term due to eddy PV fluxes, the degree to which the simple steady state approximation with down-gradient PV fluxes is valid may be tested. We have evaluated this term based on 19792010 European Centre for Medium-Range Weather Forecasts interim reanalysis (ERA-interim) data in isentropic coordinates. The primitive equation version in isentropic coordinates of the (full) enstrophy budget, as appropriate for this analysis, reads (cf. adiabatic version in Jansen and Ferrari [2013])

$$
\begin{aligned}
\bar{D}_{t}^{*} \frac{{\overline{\hat{P}^{2}}}^{*}}{2}= & \overline{\hat{S} \hat{P}}^{*}-\overline{\hat{v} \hat{P}}^{*}\left(a^{-1} \partial_{\varphi} \bar{P}^{*}\right)-\overline{\hat{Q} \hat{P}}{ }^{*} \partial_{\theta} \bar{P}^{*} \\
& -\bar{\sigma}^{-1} \partial_{y}\left(\sigma \hat{\hat{v} \frac{\hat{P}^{2}}{2}} \cos \varphi\right)-\bar{\sigma}^{-1} \partial_{\theta} \overline{\hat{Q}^{\hat{P}^{2}}}
\end{aligned}
$$

with $P \equiv(\zeta+f) / \sigma$ the (Rossby-Ertel) $\mathrm{PV}, Q$ the diabatic heating rate (in terms of potential temperature $\theta$ ), $\sigma=$ $-\left(g \partial_{p} \theta\right)^{-1}$ is the isentropic density, $y \equiv a \sin \varphi$, and

$$
\bar{D}_{t}^{*} \equiv \partial_{t}+a^{-1} \bar{v}^{*}\left(\partial_{\varphi}-\tan \varphi\right)+\bar{Q}^{*} \partial_{\theta} .
$$

In isentropic coordinates, the zonal averaging operator and the respective deviations thereof are appropriately defined using mass weighting as [e.g., Andrews, 1983b]:

$$
\hat{X} \equiv X-\bar{X}^{*}, \text { with } \quad \bar{X}^{*} \equiv \frac{\overline{\sigma X}}{\bar{\sigma}} .
$$

With the above second order balance and neglecting the vertical (cross isentropic) eddy flux terms (third and fifth terms in equation (3)), this enstrophy budget reduces to (cf. equation (1))

$$
\partial_{t} \frac{{\overline{\hat{P}^{2}}}^{*}}{2}=\overline{\hat{S}}^{*}-\overline{\hat{v}}^{*}\left(a^{-1} \partial_{\varphi} \bar{P}^{*}\right) .
$$

[12] Figure 1 (top row) shows the enstrophy tendency due to eddy PV fluxes - the term $\overline{\hat{v}}^{*}\left(a^{-1} \partial_{\varphi} \bar{P}^{*}\right)$ - as a function of latitude and potential temperature, separately for each hemisphere's winter season averaged over 1979-2010. Note the sign convention in this and all the following figures: Negative values denote down-gradient PV fluxes, which correspond to positive enstrophy tendencies, and vice versa.

[13] Eddy PV fluxes appear to be predominantly downgradient, i.e., $\overline{\hat{v}}^{*}\left(a^{-1} \partial_{\varphi} \bar{P}^{*}\right)<0$, consistent with the 
down-gradient (blue) vs. up-gradient (red) eddy PV fluxes

(a)
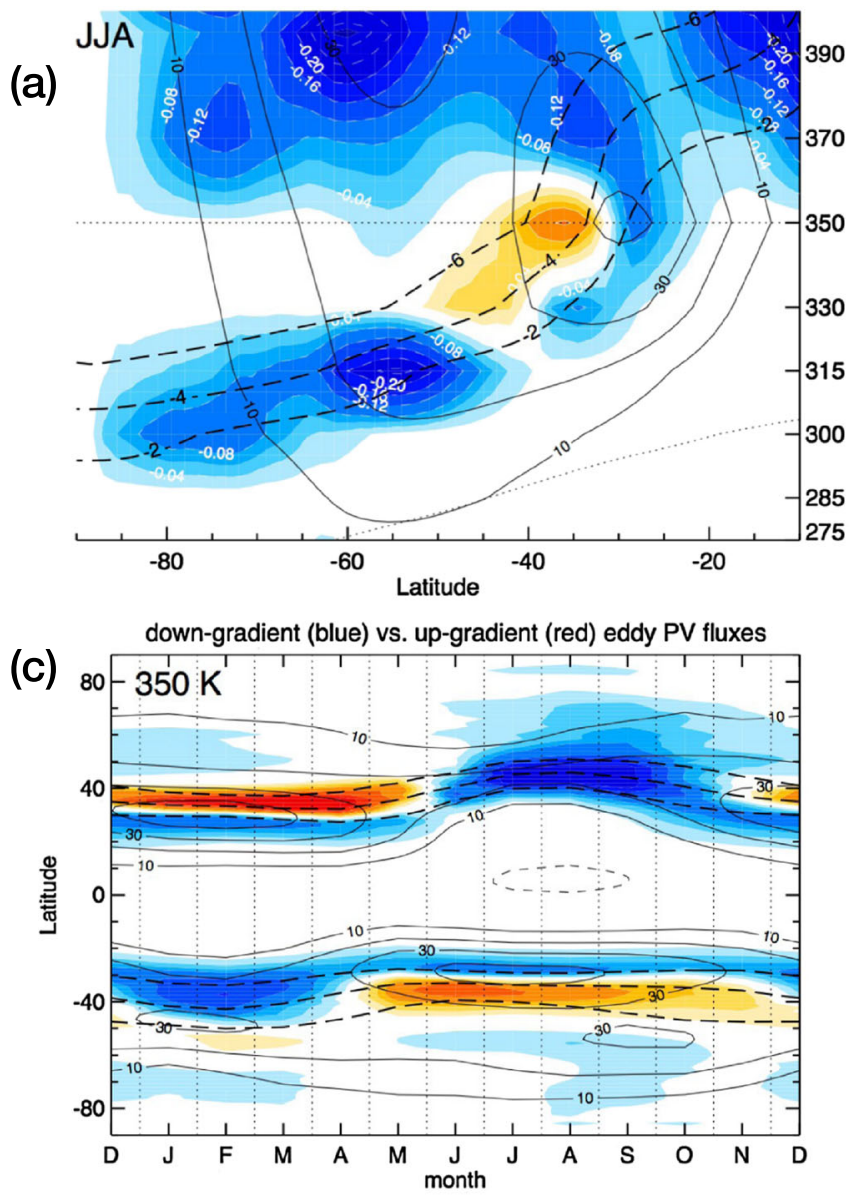
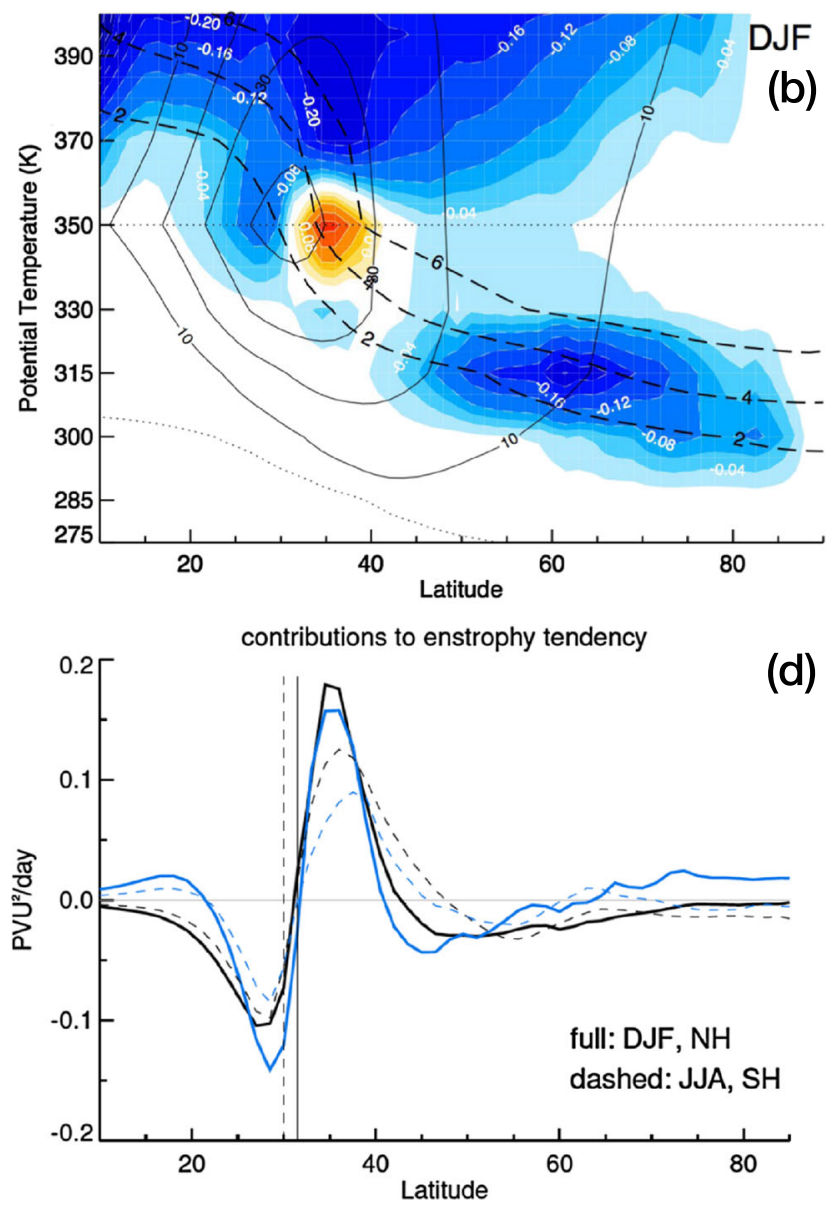

Figure 1. (top row) Seasonal mean latitude - potential temperature cross sections of the enstrophy tendency due to eddy PV fluxes (potential vorticity unit (PVU) ${ }^{2}$ /day, color shading and white contours). (a) Southern hemisphere, JJA and (b) northern hemisphere, DJF. Negative/positive values (blue/red shading) mark down-/up-gradient eddy PV fluxes and hence positive/negative enstrophy tendencies. Zonal mean zonal wind ( $\mathrm{m} / \mathrm{s}$; thin black contours), near-tropopause PV isolines ( $\mathrm{PV}= \pm 2, \pm 4, \pm 6 \mathrm{PVU}$; black dashed), and $850 \mathrm{hPa}$ pressure surface (dotted contour). The horizontal dotted line highlights the $350 \mathrm{~K}$ surface. (c) Seasonal cycle as function of latitude on the $350 \mathrm{~K}$ isentropic surface of the enstrophy tendency due to eddy PV fluxes (color shading, same contour spacing as in top row). (d) Seasonal mean of enstrophy tendency due to eddy PV fluxes (black lines, negative values mark down-gradient fluxes and hence positive enstrophy tendencies, and vice versa), and the convergence of the eddy enstrophy flux (light blue lines) as a function of latitude on the $350 \mathrm{~K}$ surface (northern hemisphere, DJF (full) and southern hemisphere, JJA (dashed)). Vertical lines mark average location of the jet maximum.

diffusive picture. A striking exception appears just poleward of the subtropical jet, where eddy PV fluxes are up-gradient in a region of strongly positive PV gradients. The strength of the eddy PV flux (related to the mean flow forcing) in these regions is small compared to other regions of down-gradient fluxes (not shown). However, as evident in Figure 1, because of the strong PV gradients near the subtropical jets, the enstrophy tendency due to the up-gradient fluxes just poleward of the subtropical jet is of similar strength compared to the tendency due to down-gradient fluxes in other regions (e.g., the region of strong mean flow forcing near the middle and polar latitude tropopause). Note that the up-gradient ( positive) eddy PV flux just poleward of the jet core represents a positive angular momentum $(\sim$ mean flow $)$ tendency which together with the down-gradient ( $\sim$ negative) eddy PV flux just equatorward of the jet core tends to shift the jet poleward. The region of up-gradient eddy PV flux has previously been pointed out by Bartels et al. [1998], based on an analysis of transient eddy PV fluxes along isentropic surfaces from operational meteorological analyses for 1984-1993 and in a general circulation model. Swanson [2001] also pointed out localized regions of strong upgradient transient eddy PV fluxes over North America and Europe. The up-gradient eddy PV flux corresponds to divergent Eliassen-Palm flux and equatorward residual flow (which curiously is evident in many previous studies) [e.g., Edmon et al., 1980; Karoly, 1982], in all respects an anomalous atmospheric region.

[14] The region of up-gradient eddy PV flux appears to be concentrated on the $350 \mathrm{~K}$ isentropic surface. Figure 1c 
shows the seasonal cycle of the term $\overline{\hat{v}}^{*}\left(a^{-1} \partial_{\varphi} \bar{P}^{*}\right)$ as a function of latitude on the $350 \mathrm{~K}$ isentropic surface. The region of up-gradient eddy $\mathrm{PV}$ fluxes exists throughout the cold season in each hemisphere, with a dipole of downgradient fluxes on the equatorward flank and up-gradient fluxes on the poleward flank consistently straddling the subtropical jet. A similar, although weaker, feature appears in the Southern hemisphere's summer season.

\section{Up-Gradient Eddy Fluxes of Potential Vorticity and Poleward Jet Displacements}

[15] The region of up-gradient eddy fluxes of PV is inconsistent with the diffusive picture of the general circulation; it formally corresponds to a negative eddy diffusivity in the flux-gradient relation ( $\sim$ antidiffusion). It is also inconsistent with the simple steady state balance suggested by equation (4), assuming $\hat{S}$ represents dissipative processes with $\overline{\hat{S}}^{*}<0$. Other terms in the enstrophy budget must therefore play an important role to balance the term due to up-gradient eddy fluxes of PV. One candidate is the forcing term due to the convergence of enstrophy fluxes, which alters the budget in the following way in the QG system:

$$
\partial_{t} \frac{\overline{q^{\prime 2}}}{2}=\overline{S^{\prime} q^{\prime}}-\overline{v^{\prime} q^{\prime}} \partial_{y} \bar{q}-\partial_{y} \overline{v^{\prime} \frac{q^{\prime 2}}{2}} ;
$$

and in the following way in the isentropic coordinate primitive equations:

$$
\partial_{t} \frac{{\overline{\hat{P}^{2}}}^{*}}{2} \approx \overline{\hat{S} \hat{P}}^{*}-\overline{\hat{v} \hat{P}}^{*}\left(a^{-1} \partial_{\varphi} \bar{P}^{*}\right)-\bar{\sigma}^{1} \partial_{y}\left(\sigma \hat{\hat{v} \frac{\hat{P}^{2}}{2}} \cos \varphi\right) .
$$

The extra term is not necessarily inconsistent with the QG approximation or the assumption that the waves/eddies are quasi-adiabatic. But given that it is $\mathcal{O}(3)$ in the perturbation, it is inconsistent with the assumption of quasi-linear waves, i.e., it represents nonlinear effects. The enstrophy flux term has previously been found to be important in the context of interaction of eddies with the Hadley cell [Held and Phillips, 1990].

[16] Figure 1d shows both eddy forcing terms in the above enstrophy budget (due to eddy PV fluxes and eddy enstrophy fluxes) along the $350 \mathrm{~K}$ isentropic surface during each hemisphere's winter season. Overall, there is a remarkable balance between these two terms, implying a net zero leading order eddy contribution to the enstrophy budget and suggesting other terms in the full budget (equation (3)) to be less important. Somewhat surprisingly, dissipation does not appear to represent a dominant process in the (local) enstrophy balance, implying the simple balance in equations (2) and (4) to not hold. The qualitative balance between the two eddy terms is especially remarkable, given the highly derived nature of the budget terms combined with the fact that such budgets are often hard to balance using off-line analysis data as used here. Apparently, the up-gradient eddy flux of PV on the poleward flank of the subtropical jet (representing negative wave activity tendency, i.e., local perturbation decay) is largely balanced by convergence of enstrophy flux (representing positive wave activity tendency, i.e., local perturbation growth). The quasilinear description of eddies appears inadequate over much of the subtropics and midlatitudes along the $350 \mathrm{~K}$ isentropic surface. This may be expected given this region of the atmosphere is dominated by finite-amplitude wave perturbations and associated wave breaking. Furthermore, the balance is dominated by contributions due to conservative effects with nonconservative effects (such as dissipation) of secondary importance.

[17] Wave growth and subsequent breaking tends to be associated with positive enstrophy tendencies (i.e., increases in PV variance). Figure 1d suggests finite-amplitude effects to be important for wave-breaking events near the subtropical jet. The region just equatorward of the subtropical jet appears consistent with these suppositions: The positive enstrophy tendency due to the down-gradient eddy PV fluxes is largely balanced by the divergence of the enstrophy flux there. This divergence results in significant poleward enstrophy fluxes across the mean latitude of the jet maximum. Just poleward of the jet maximum, the convergence of enstrophy flux (a positive enstrophy tendency) is balanced by the contribution due to up-gradient PV fluxes (a negative enstrophy tendency). Enstrophy growth due to the down-gradient PV fluxes equatorward of the jet is therefore linked with enstrophy decay due to the up-gradient PV fluxes poleward of the jet through fluxes of enstrophy between the two regions.

[18] The enstrophy flux represents a triple correlation, hence, it can by definition only be nonzero for interactions among different parts of the wave spectrum. A more detailed analysis (to be published elsewhere) as to which part of the wave spectrum contributes to the terms shown in Figure 1d reveals that the down-gradient PV fluxes on the equatorward flank of the jet are primarily due to synoptic-scale waves (wave numbers 3-7), whereas the up-gradient PV fluxes on the poleward flank of the jet are primarily due to planetary-scale waves (wave numbers 1-2). Thus, the poleward enstrophy flux across the mean jet latitude appears to largely represent generation of planetary wave activity at the expense of synoptic-scale wave activity.

[19] The above suggests the following typical chain of events: Enstrophy (1) grows through (synoptic-scale) wave breaking on the equatorward flank of the subtropical jet, (2) is fluxed poleward across the mean latitude of the jet maximum, and (3) decays (at planetary scales) on the poleward flank of the jet. That is, the up-gradient PV flux is a result of the eddy life cycle along the subtropical jet, which importantly includes both a temporal and a spatial component, as well as interactions across the wave spectrum.

[20] However, if wave breaking is associated with downgradient PV fluxes, how can it result in up-gradient PV fluxes on the poleward flank of the subtropical jet?

[21] As discussed in the previous section, up-gradient eddy PV fluxes are produced temporarily during wave decay. As long as wave decay takes place at the same latitude as the preceding wave growth and wave breaking, the up-gradient PV fluxes are overwhelmed by the down-gradient PV fluxes. However, if wave decay takes place at a different latitude than the preceding wave growth and breaking, due to latitudinal progression of the underlying wave guide (the jet core), a portion of the up-gradient PV flux may locally remain after time averaging. Figure 1 shows that the structure of eddy PV fluxes around the jet core tend to force the jet poleward, consistent with the poleward propagation of the jet due to wave breaking along its axis [e.g., Feldstein, 1998; Lee et al., 2007].

[22] In Figure 2, we show that individual events of enhanced wave activity (which take place on daily to weekly 

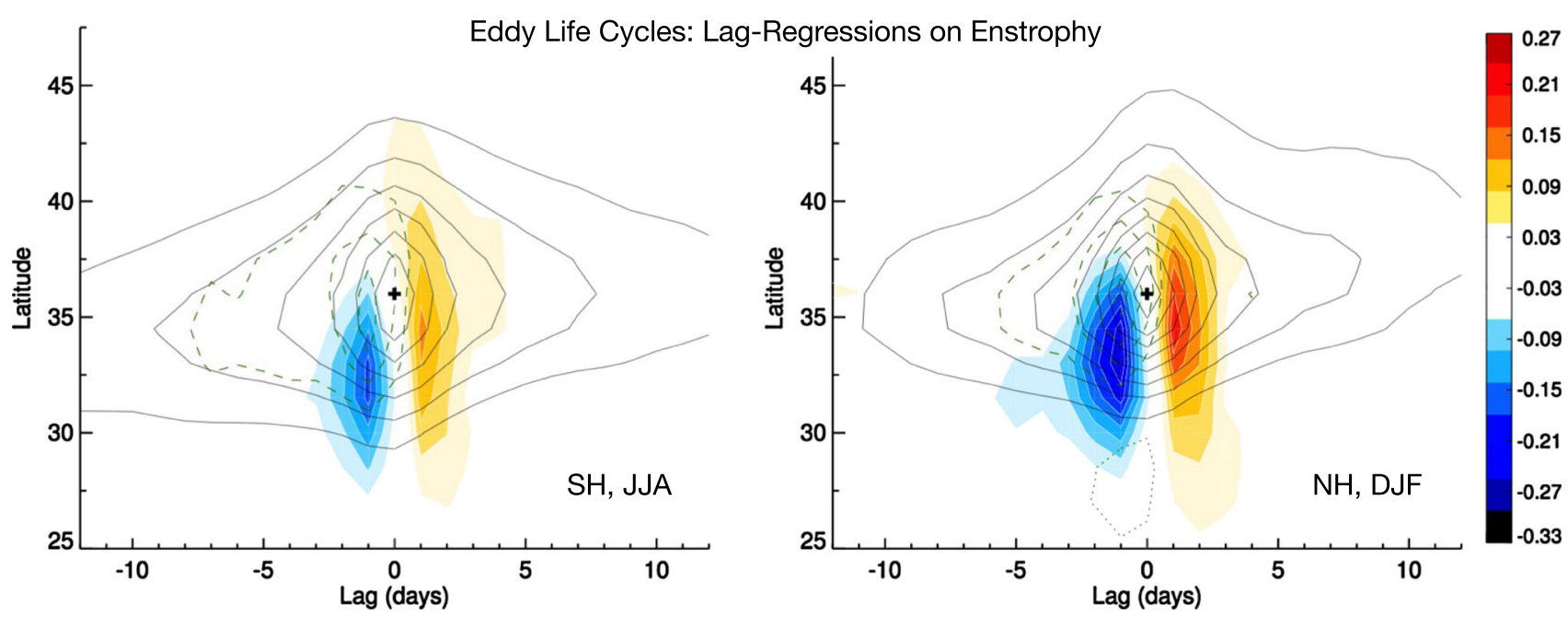

Figure 2. Regressions as a function of lag and latitude on enstrophy at $36^{\circ} \mathrm{N} / \mathrm{S}$ (marked at lag 0 by the plus sign) along the $350 \mathrm{~K}$ isentropic surface ((left) southern hemisphere, JJA and (right) northern hemisphere, DJF). Black contours show enstrophy (contour spacing 0.1 ), color contours show the enstrophy tendency due to eddy PV fluxes (negative values mark down-gradient fluxes and hence positive enstrophy tendencies, and vice versa), and green contours show the enstrophy flux (poleward dashed, equatorward dotted, contour spacing 0.2 , no zero contour). All regression values are standardized.

time scales) are associated with a poleward shift in the region of largest enstrophy anomalies that leads to a region of net poleward (up-gradient) PV fluxes. The results in Figure 2 show the typical evolution of enhancements in enstrophy in ERA-interim near the subtropical jet using linear regression analysis based on deseasonalized daily anomalies (rather than the long-term mean) of the zonal-mean circulation. We associate enhanced enstrophy with wave breaking. Figure 2 shows lag regressions onto deseasonalized daily enstrophy anomalies at the average long-term mean location of the up-gradient PV flux $\left(36^{\circ} \mathrm{N} / \mathrm{S}\right)$ for both hemisphere's winter seasons. A clear poleward progression of enstrophy is evident on a time scale of $\sim 10$ days (black contours). Analyzing regressions of the zonal wind as well as the meridional PV gradient (both zonal mean and zonally asymmetric components, not shown) confirms that this poleward progression is consistent with the poleward displacement of the jet during wave breaking, which involves modifications in the meridional tilt of the jets.

[23] The results in Figure 2 reveal that periods of enhanced enstrophy near the core of the jet are associated with (1) anomalous equatorward (down-gradient) eddy PV fluxes before the peak in enstrophy, corresponding to a positive enstrophy tendency (consistent with local wave growth) and (2) anomalous poleward (up-gradient) eddy PV fluxes after the peak in enstrophy, corresponding to a negative enstrophy tendency (consistent with local wave decay). Importantly, the up-gradient PV fluxes at positive lags are displaced poleward relative to the down-gradient PV fluxes at negative lags, as hypothesized above. That is, wave decay takes place poleward of the originating latitude of wave growth and subsequent wave breaking. As can be further seen in Figure 2, the enstrophy that is generated at negative lags is fluxed poleward following the jet displacement. Averaging in time and latitude over the entire event gives net down-gradient eddy PV fluxes (positive enstrophy tendency) as is evident by inspecting the durations and strengths of the opposite-signed tendencies at negative and positive lags, respectively. That is, applied to the jet region as a whole, the wave-mean flow interaction acts to reduce angular momentum, as expected. However, this does not hold locally near the jet core due to the finite meridional extent of the eddy life cycle and the associated finite meridional displacement of the jet. Note that the depicted enstrophy anomalies represent integrated tendencies; they are quantitatively very accurately reproduced by the integrated tendencies due to eddy PV and enstrophy fluxes, confirming that diabatic or dissipative processes are less important.

[24] Similar to the remarks about the climatological structure in Figure 1d, the down-gradient PV fluxes at negative lags in Figure 2 are primarily due to synoptic-scale waves (wave numbers 3-7), whereas the up-gradient PV fluxes at positive lags are primarily due to planetary-scale waves (wave numbers 1-2). Both parts of the wave spectrum are linked through the enstrophy flux term, which appears to predominantly transfer enstrophy from synoptic to planetary scales during the life cycle. This link together with the up-gradient PV fluxes therefore indicates a source of planetary-scale wave activity that could then vertically propagate away from the $350 \mathrm{~K}$ surface.

[25] The planetary-scale ( quasi-stationary) part also represents zonal asymmetries, e.g., the Pacific and Atlantic jets and their meridional tilts in the Northern Hemisphere; enhancements and reductions in planetary-scale wave amplitude correspond to enhancements and reductions in these jet tilts. The eddy life cycles shown in Figure 2 show a remarkable similarity between the two hemispheres, suggesting basic state zonal asymmetries to be of secondary importance.

\section{Summary}

[26] The zonal-mean, time-mean atmospheric eddy fluxes of PV along isentropic surfaces are generally believed to be down-gradient throughout the extratropical free atmosphere. Given that the background PV gradients are predominantly 
positive, these down-gradient PV fluxes tend to reduce the angular momentum of the mean flow. Here we have identified a robust, localized region of significant up-gradient eddy PV fluxes on the poleward flank of the subtropical jet core. The up-gradient PV fluxes peak near the $350 \mathrm{~K}$ isentrope (i.e., near $200 \mathrm{hPa}$ ) during the winter and spring seasons in both hemispheres. They exist in a region of strong and positive background PV gradient and thus enhance the angular momentum of the mean flow. The region of upgradient (notionally antidiffusive) eddy PV flux is in conflict with the (macro)turbulence approach to the eddy fluxes, at least applied locally.

[27] Analysis of the potential enstrophy budget suggests that the region of up-gradient PV fluxes represents local wave decay and is maintained by poleward enstrophy fluxes. We provide evidence that the poleward migration of the jet acts to shift the latitude of wave decay poleward of the latitude of wave growth and breaking, thus giving rise to a region of down-gradient PV fluxes on the equatorward flank of the subtropical jet and a region of up-gradient PV fluxes on its poleward flank. The net PV fluxes are down-gradient when averaged over both the equatorward and poleward flanks of the jet, and thus, the PV fluxes are diffusive when averaged over the entire jet region. This net down-gradient bulk PV flux is then balanced by dissipation. The flux-gradient relation is apparently not applicable near the jet core because of the finite meridional extent of the wave-breaking region.

[28] A primary conclusion of this study is that finiteamplitude effects (related to variance fluxes) play a central role for upper level eddy-mean flow interactions, in particular, near the subtropical jet. Our results thus undermine the usual assumption, at least locally, that PV fluxes are balanced by dissipation. A finite-amplitude eddy-mean flow interaction formalism, such as recently introduced by Nakamura and Zhu [2010], may therefore help to further elucidate the dynamics of the up-gradient PV fluxes.

[29] Because up-gradient PV fluxes imply an increase in atmospheric angular momentum, the region of up-gradient PV fluxes reflects a seemingly fundamental aspect of jet dynamics. Wave-driven shifts in the jets play a fundamental role in climate variability across a range of timescales. And simulations with an idealized GCM suggest that the up-gradient eddy fluxes of PV can become relatively more important in warmer climates (P. O'Gorman, personal communication, 2013), with implications not only for the structure of the atmospheric circulation but for the surface flow as well. The implications of the up-gradient PV fluxes for climate variability and change remain to be determined.

[30] Acknowledgments. We thank the thoughtful and stimulating reviewer comments. Useful discussions with Dieter Peters, Tiffany Shaw, Malte Jansen, Paul O'Gorman, and Joseph Kidston. Gang Chen's suggestion to analyze enstrophy fluxes is greatly appreciated. ERA-interim data were provided by ECMWF. TB and DWJT acknowledge support by the NSF Climate Dynamics Program.

[31] The Editor thanks Walter Robinson and Raymond Plumb for their assistance in evaluating this paper.

\section{References}

Andrews, D. G. (1983a), A conservation law for small-amplitude quasigeostrophic disturbances on a zonally asymmetric basic flow, J. Atmos. Sci., 40, 85-90.

Andrews, D. G. (1983b), A finite-amplitude Eliassen-Palm theorem in isentropic coordinates, J. Atmos. Sci., 40, 1877-1883.
Andrews, D. G., J. R. Holton, and C. B. Leovy (1987), Middle Atmosphere Dynamics, 489 pp., Academic Press, San Diego, Calif.

Bartels, J., D. Peters, and G. Schmitz (1998), Climatological Ertel's potential-vorticity flux and mean meridional circulation in the extratropical troposphere-Lower stratosphere, Ann. Geophys., 16, 250-265.

Butler, A. H., D. W. J. Thompson, and T. Birner (2011), Isentropic slopes, downgradient eddy fluxes, and the extratropical atmospheric circulation response to tropical tropospheric heating, J. Atmos. Sci., 68, 2292-2305.

Defant, A. (1921), Die Zirkulation der Atmosphäre in den gemässigten Breiten der Erde. Grundzüge einer Theorie der Klimaschwankungen, Geograf. Ann., 3, 209-266.

Edmon, H. J., B. J. Hoskins, and M. E. McIntyre (1980), Eliassen-Palm cross sections for the troposphere, J. Atmos. Sci., 37, 2600-2616.

Feldstein, S. B. (1998), An observational study of the intraseasonal poleward propagation of zonal mean flow anomalies, J. Atmos. Sci., 55, 2516-2529.

Green, J. S. A. (1970), Transfer properties of the large-scale eddies and the general circulation of the atmosphere, Q. J. R. Meteorol. Soc., 96, $157-185$.

Haynes, P., J. Scinocca, and M. Greenslade (2001), Formation and maintenance of the extratropical tropopause by baroclinic eddies, Geophys. Res. Lett., 28, 4179-4182.

Held, I. M. (1999), The macroturbulence of the troposphere, Tellus, Ser. B, $51,59-70$.

Held, I. M. (2000), The general circulation of the atmosphere, Lecture Notes, Woods Hole GFD Summer School.

Held, I. M., and B. J. Hoskins (1985), Large-scale eddies and the general circulation of the troposphere, in Advances in Geophysics, vol. 28, edited by B. Saltzman, pp. 3-31, Academic Press, New York.

Held, I. M., and P. J. Phillips (1990), A barotropic model of the interaction between the Hadley cell and a Rossby wave, J. Atmos. Sci., 47, $856-869$

Hoskins, B. J. (1983), Modelling of the transient eddies and their feedback on the mean flow, in Large-Scale Dynamical Processes in the Atmosphere, edited by B. Hoskins and R. Pearce, pp. 169-199, Academic Press, New York.

Hoskins, B. J., M. E. McIntyre, and A. W. Robertson (1985), On the use and significance of isentropic potential vorticity maps, $Q$. J. R. Meteorol. Soc., 111, 877-946.

Jansen, M., and R. Ferrari (2013), The vertical structure of the eddy diffusivity and the equilibration of the extratropical atmosphere, J. Atmos. Sci., 70, 1456-1469.

Jeffreys, H. (1926), On the dynamics of geostrophic winds, $Q . J . R$. Meteorol. Soc., 51, 85-101.

Karoly, D. J. (1982), Eliassen-Palm cross sections for the northern and southern hemispheres, J. Atmos. Sci., 39, 178-182.

Lee, S., S.-W. Son, K. Grise, and S. B. Feldstein (2007), A mechanism for the poleward propagation of zonal mean flow anomalies, J. Atmos. Sci., $64,849-868$.

Lorenz, D. J., and D. L. Hartmann (2001), Eddy-zonal flow feedback in the southern hemisphere, J. Atmos. Sci., 58, 3312-3327.

McIntyre, M. E. (2000), On global-scale atmospheric circulations, in Perspectives in Fluid Dynamics, edited by G. K. Batchelor, H. K. Moffatt, and M. G. Worster, pp. 557-624, Cambridge Univ. Press, Cambridge, U. K.

McIntyre, M. E. (2008), Potential-vorticity inversion and the waveturbulence jigsaw: Some recent clarifications, Adv. Geosci., 15, 47-56.

Nakamura, N., and D. Zhu (2010), Finite-amplitude wave activity and diffusive flux of potential vorticity in eddy-mean flow interaction, J. Atmos. Sci. 67, 2701-2716.

Plumb, R. A. (1979), Eddy fluxes of conserved quantities by smallamplitude waves, J. Atmos. Sci., 36, 1699-1704.

Randel, W. J., and I. M. Held (1991), Phase speed spectra of transient eddy fluxes and critical layer absorption, J. Atmos. Sci., 48, 688-697.

Rhines, P. B., and W. R. Young (1982), Homogenization of potential vorticity in planetary gyres, J. Fluid Mech., 122, 347-367.

Robinson, W. A. (2000), A baroclinic mechanism for the eddy feedback on the zonal index, J. Atmos. Sci., 57, 415-422.

Schneider, T. (2004), The tropopause and the thermal stratification in the extratropics of a dry atmosphere, J. Atmos. Sci., 61, 1317-1340.

Sobel, A. H. (1999), Diffusion versus nonlocal models of stratospheric mixing, in theory and practice, J. Atmos. Sci., 56, 2571-2584.

Swanson, K. L. (2001), Upper-tropospheric potential vorticity fluctuations and the dynamical relevance of the time mean, J. Atmos. Sci., 58, 1815-1826.

Taylor, G. I. (1914), Eddy motion in the atmosphere, Proc. London Math. Soc., 2, 196-211.

Thompson, D. W. J., and T. Birner (2012), On the linkages between the tropospheric isentropic slope and eddy fluxes of heat during northern hemisphere winter, J. Atmos. Sci., 69, 1811-1823.

Vallis, G. K. (2006), Atmospheric and Oceanic Fluid Dynamics, 745 pp., Cambridge Univ. Press, Cambridge, U. K. 